\title{
Photoacoustic computed tomography without accurate ultrasonic transducer responses
}

Qiwei Sheng, Kun Wang, Jun Xia, Liren Zhu, Lihong V. Wang, et al.

Qiwei Sheng, Kun Wang, Jun Xia, Liren Zhu, Lihong V. Wang, Mark A. Anastasio, "Photoacoustic computed tomography without accurate ultrasonic transducer responses," Proc. SPIE 9323, Photons Plus Ultrasound: Imaging and Sensing 2015, 932313 (11 March 2015); doi: 10.1117/12.2081056

SPIE. Event: SPIE BiOS, 2015, San Francisco, California, United States 


\title{
Photoacoustic computed tomography without accurate ultrasonic transducer responses
}

\author{
Qiwei Sheng $^{a}$, Kun Wang ${ }^{a}$, Jun $\mathrm{Xia}^{b}$, Liren Zhu ${ }^{a}$, Lihong V. Wang ${ }^{a}$, and Mark A. Anastasio ${ }^{a}$ \\ ${ }^{a}$ Department of Biomedical Engineering, Washington University in St. Louis, \\ St. Louis, MO 63130 \\ ${ }^{b}$ Department of Biomedical Engineering, The University at Buffalo, Buffalo, NY 14260
}

\begin{abstract}
Photoacoustic computed tomography (PACT) holds great promise for biomedical imaging. When the imaging system employs conventional piezoelectric ultrasonic transducers, the ideal photoacoustic (PA) signals are degraded by the transducers' acousto-electric impulse responses (EIRs) during the measurement process. If unaccounted for, this can degrade the accuracy of the reconstructed image. In principle, the effect of the EIRs on the measured PA signals can be ameliorated via deconvolution; images can be reconstructed subsequently by application of a reconstruction method that assumes an idealized EIR. Alternatively, the effect of the EIR can be incorporated into an imaging model and implicitly compensated for during reconstruction. In either case, the efficacy of the correction can be limited by errors in the assumed EIRs. In this work, a joint optimization approach to PACT image reconstruction is proposed for mitigating errors in reconstructed images that are caused by use of an inaccurate EIR. A variable projection method is employed to refine the measured EIR during the process of reconstructing the sought-after absorbed optical energy density. Computer-simulation and experimental studies are conducted to investigate the numerical properties of the method and demonstrate its value for mitigating image distortions and enhancing the visibility of fine structures.
\end{abstract}

\section{INTRODUCTION}

In PACT, ${ }^{1-5}$ tissues are illuminated by short laser pulses to generate internal broadband photoacoustic wavefields via the photoacoustic effect. ${ }^{1,2}$ From the measurements of the propagated acoustic wavefields outside the object, an image reconstruction method can be employed to estimate the absorbed optical energy density within the tissue.

A variety of PACT imaging systems have been developed. When the system employs piezoelectric transducers, the PA signals at the transducer locations are convolved with the transducers' acousto-electric impulse responses (EIRs) to generate the output digital signals. If unaccounted for, this degradation of the measurement data will result in a modulation of the spatial frequency components of the estimated absorbed optical energy density distribution. ${ }^{6}$ In principle, if the EIR is known accurately, the effect of the EIRs on the PA signals can be removed via deconvolution or compensated for implicitly during image reconstruction by use of an imaging model incorporating the EIR..$^{7-9}$ However, acquiring an accurate measurement of the EIR can be difficult. ${ }^{10}$ Furthermore, there are typically hundreds of individual elements with unique EIRs of their own in an transducer array, and it can be an arduous task to characterize each EIR.

The purpose of this study is to develop a joint optimization approach to simultaneously estimate the absorbed optical energy density and the EIR of the transducer array. To achieve this, a variable projection method ${ }^{11-13}$ is employed to refine the measured EIR during the process of reconstruction.

(Send correspondence to:)

Mark Anastasio: E-mail: anastasio@wustl.edu

Photons Plus Ultrasound: Imaging and Sensing 2015, edited by Alexander A. Oraevsky, Lihong V. Wang

Proc. of SPIE Vol. 9323, 932313 - ( 2015 SPIE · CCC code: $1605-7422 / 15 / \$ 18$

doi: $10.1117 / 12.2081056$

Proc. of SPIE Vol. $9323932313-1$ 


\section{BACKGROUND}

A discrete-to-discrete imaging model ${ }^{1,2,5,14,15}$ for PACT can be established as

$$
\mathbf{u}=\mathbf{H}(\mathbf{h}) \boldsymbol{\theta}
$$

where $\boldsymbol{\theta}$ is a finite-dimensional representation of the sought-after object, i.e., absorbed optical energy density distribution, $\mathbf{u}$ denotes the measured data vector, $\mathbf{h}$ denotes the vector of EIR samples, and $\mathbf{H}$ denotes a system matrix that maps the absorbed optical energy density distribution to the measured data vector. Generally, the number of unknowns in $\mathbf{h}$ is significantly smaller than the number of unknowns in $\boldsymbol{\theta}$. The notation $\mathbf{H}(\mathbf{h})$ indicates the dependence of $\mathbf{H}$ on $\mathbf{h}$. A conventional penalized least squares image reconstruction method seeks the solution

$$
\hat{\boldsymbol{\theta}}=\underset{\boldsymbol{\theta} \geq 0}{\operatorname{argmin}}\|\mathbf{u}-\mathbf{H}(\mathbf{h}) \boldsymbol{\theta}\|^{2}+\lambda R_{1}(\boldsymbol{\theta}) .
$$

Here $R_{1}(\boldsymbol{\theta})$ denotes the penalty term, whose impact is controlled by the regularization parameter $\lambda$.

A standard gradient descent algorithm solves Eqn. (2) iteratively by updating the estimate of $\boldsymbol{\theta}$ by taking a small step along the direction opposite the gradient of the cost function. The step size is commonly determined by using a line search method. A conventional iterative reconstruction method based upon a gradient descent algorithm is described in Alg. 1. In Alg. $1, \mathcal{P}_{\mathrm{c}}$ denotes the operator that projects all negative values to 0 , $\gamma^{k}$ is

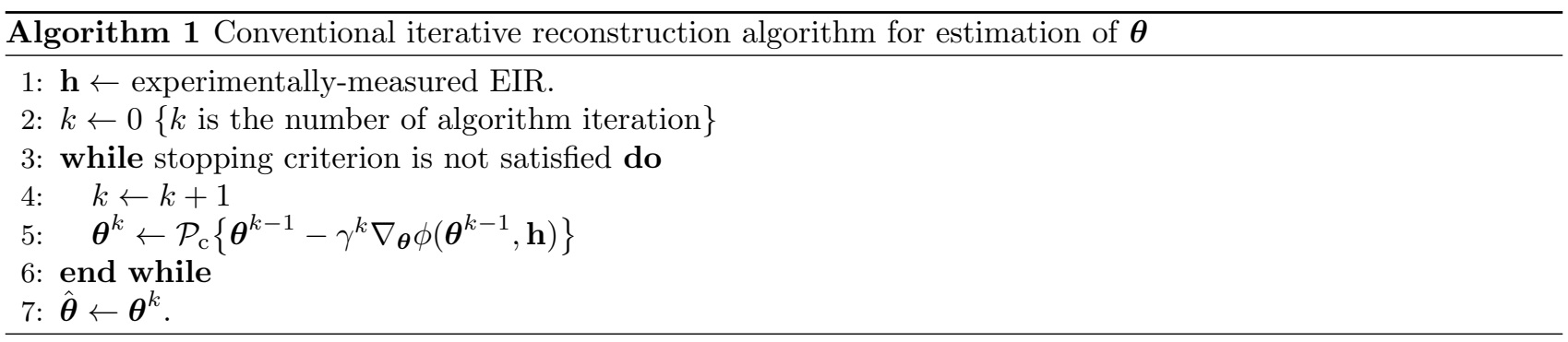

the step size, $\nabla_{\boldsymbol{\theta}}$ is the gradient operator with respect to $\boldsymbol{\theta}$, and $\phi(\boldsymbol{\theta}, \mathbf{h})=\|\mathbf{u}-\mathbf{H}(\mathbf{h}) \boldsymbol{\theta}\|^{2}+\lambda R_{1}(\boldsymbol{\theta})$. Note that the EIR $\mathbf{h}$ is fixed in the conventional iterative reconstruct method, and therefore errors in $\mathbf{h}$ could be propagated into the sought-after object $\boldsymbol{\theta}$.

\section{JOINT RECONSTRUCTION}

In order to mitigate errors in the reconstructed images, we formulate the image reconstruction problem as a joint numerical optimization problem

$$
(\hat{\boldsymbol{\theta}}, \hat{\mathbf{h}})=\underset{\boldsymbol{\theta} \geq 0, \mathbf{h}}{\operatorname{argmin}} \varphi(\boldsymbol{\theta}, \mathbf{h})
$$

where the cost function $\varphi(\boldsymbol{\theta}, \mathbf{h})$ is defined as

$$
\varphi(\boldsymbol{\theta}, \mathbf{h}) \equiv\|\mathbf{u}-\mathbf{H}(\mathbf{h}) \boldsymbol{\theta}\|^{2}+\lambda R_{1}(\boldsymbol{\theta})+\alpha R_{2}(\mathbf{h}) .
$$

Here, $R_{1}(\boldsymbol{\theta})$ and $R_{2}(\mathbf{h})$ represent penalty terms, whose impacts are controlled by the regularization parameters $\lambda$ and $\alpha$, respectively.

\section{VARIABLE PROJECTION METHOD}

A variable projection method is employed to reformulate the minimization problem (3). The variable projection method exploits the separable structure of the image model (1) by taking advantage of the fact that Eqn. (1) is bilinear with respect to $(\boldsymbol{\theta}, \mathbf{h})$ and $\mathbf{h}$ contains relatively few unknowns compared to $\boldsymbol{\theta}$ such that a closed form solution is possible when $\boldsymbol{\theta}$ is given. Instead of explicitly separating the variables $\boldsymbol{\theta}$ and $\mathbf{h}$ as in coordinate 
descent, the variable projection method implicitly eliminates the variable $\mathbf{h}$ to obtain a reduced problem that depends only on $\boldsymbol{\theta}$

$$
\min _{\boldsymbol{\theta} \geq 0} \varphi(\boldsymbol{\theta}, \mathbf{h}(\boldsymbol{\theta}))
$$

where $\mathbf{h}(\boldsymbol{\theta})$ is a solution of

$$
\min _{\mathbf{h}} \varphi(\boldsymbol{\theta}, \mathbf{h}) .
$$

To compute the gradient of the reduced cost function for the gradient descent algorithm, it is useful to note that

$$
\nabla_{\boldsymbol{\theta}} \varphi(\boldsymbol{\theta}, \mathbf{h}(\boldsymbol{\theta}))=\left.\nabla_{\boldsymbol{\theta}} \varphi(\boldsymbol{\theta}, \mathbf{h})\right|_{\mathbf{h}=\mathbf{h}(\boldsymbol{\theta})},
$$

which simplifies the gradient calculation; the gradient computation prescribed by Eqn. (7) is identical to that employed by the standard gradient descent methods for penalized least squares image reconstruction method. A complete description of the variable projection method is given in Alg. 2.

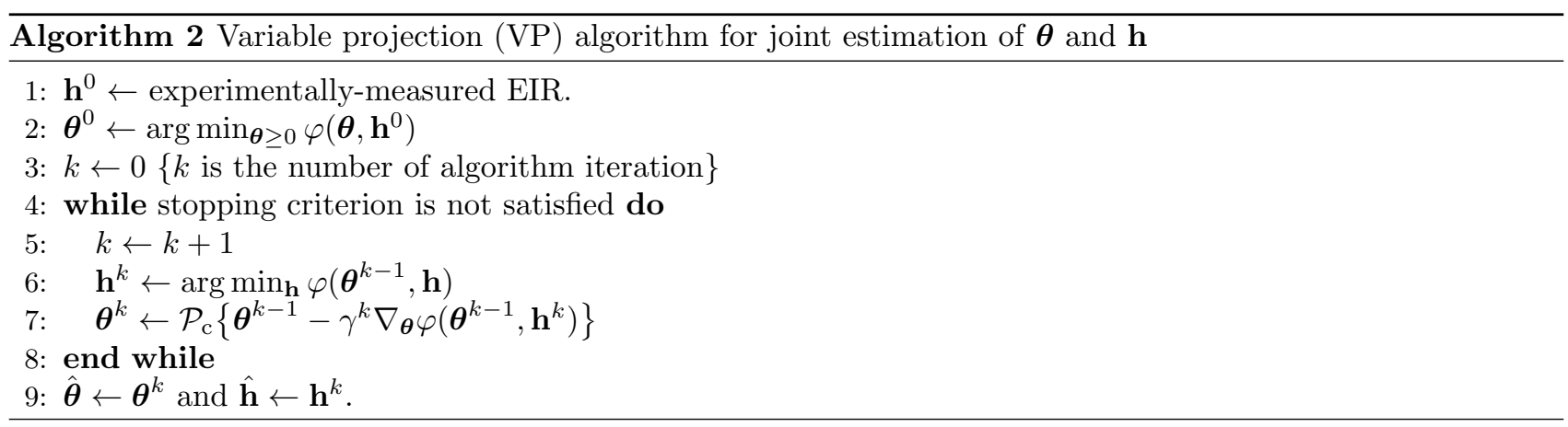

\section{DESCRIPTION OF COMPUTER-SIMULATION STUDIES}

Computer-simulation studies were conducted to demonstrate the use of the proposed joint reconstruction approach and the variable projection (VP) method.

\subsection{Generation of pressure data}

A two-dimensional (2D) numerical phantom was employed to represent the absorbed optical energy density distribution as shown in Fig. 1a. The phantom was contained in a region of $22.0 \times 22.0 \mathrm{~mm}^{2}$ and consisted of

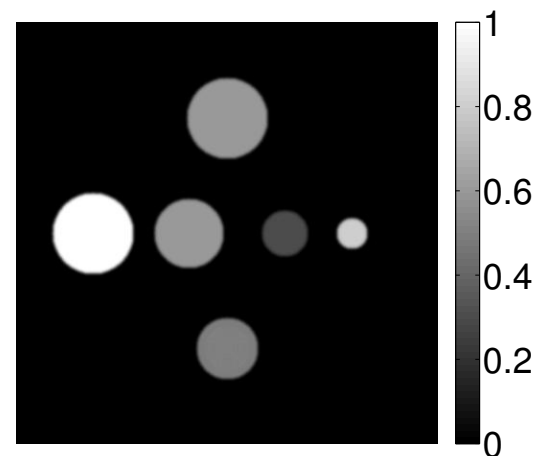

(a) Phantom

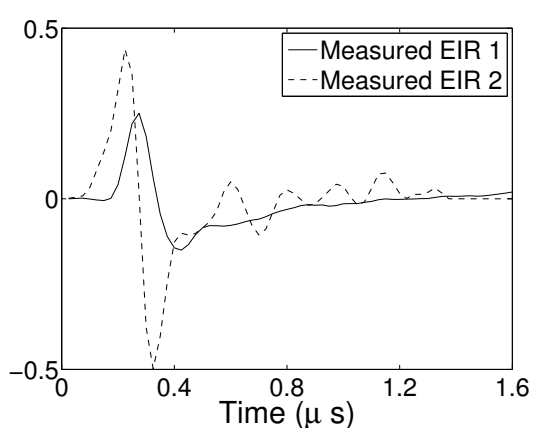

(b) EIRs

Figure 1: (a) A absorbed energy density phantom. (b) True EIR and inaccurate EIR.

six disks with various radii and absorbed optical energy densities. We employed a $2 \mathrm{D}$ ring-shaped transducer array of radius $25 \mathrm{~mm}$. The transducer array consisted of 128 uniformly-distributed detecting elements each of 
which sampled at $20 \mathrm{MHz}$. The speed-of-sound was assumed to be constant and set at $c_{0}=1.5 \mathrm{~mm} / \mu \mathrm{s}$. At each transducer, a data vector was formed by use of the C-D imaging model, ${ }^{9}$ and therefore no inverse crime was committed. We calculated 600 equally spaced temporal samples over the interval $[10,25) \mu \mathrm{s}$. Subsequently, the noiseless data vector $\mathbf{u}_{q}$ was obtained by convolving the pressure data with EIR-1 in Fig. 1b. To the noise-free data set, we added $3 \%$ Gaussian white noise to simulate noise-contaminated measurements.

\subsection{Image reconstruction methods}

From the simulated noiseless and noisy data, images were reconstructed by solving the minimization problem in Eqn. (3) by use of Algorithm 2. Conventional quadratic smoothness penalties were employed:

$$
\begin{aligned}
& R_{1}(\boldsymbol{\theta})=\sum_{n=0}^{N-1} \sum_{k \in \mathcal{N}_{n}}\left([\boldsymbol{\theta}]_{n}-[\boldsymbol{\theta}]_{k}\right)^{2} \\
& R_{2}(\mathbf{h})=\sum_{i=0}^{I-2}\left([\mathbf{h}]_{i+1}-[\mathbf{h}]_{i}\right)^{2}
\end{aligned}
$$

where $\mathcal{N}_{n}$ is the index set of four neighboring pixels of the $n$-th pixel.

The reconstructed image was represented by $440 \times 440$ pixels. The initial guess of the EIR employed in the VP algorithm was different than the EIR that was assumed when generating the simulated data. This served to simulate a situation in which an experimentally measured EIR contained errors. Algorithm 2 was terminated after 500 iterations since it was observed that the changes in the reconstructed images with more iterations were negligible. EIR-2 in Fig. 1b was employed to initialize the VP algorithm. For comparison, we also reconstructed images by use of a conventional iterative image reconstruction method that considered the EIR to be fixed. The accuracy of the reconstructed images was quantified by their root-mean-square error (RMSE) from the numerical phantom.

\section{NUMERICAL RESULTS}

The images reconstructed by use of the conventional iterative method and the VP method from noise-free data are shown in Fig. 2a and Fig. 2b, respectively, and their profiles are plotted in Fig. 2c. As confirmed by

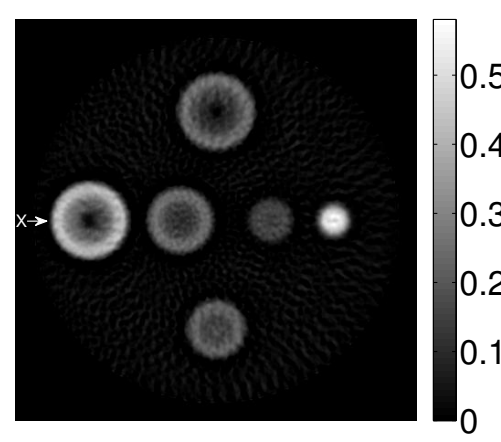

(a)

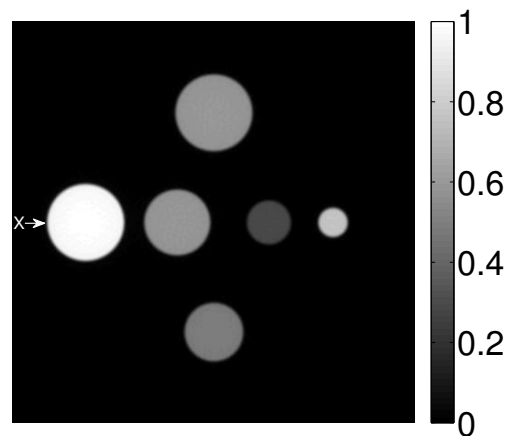

(b)

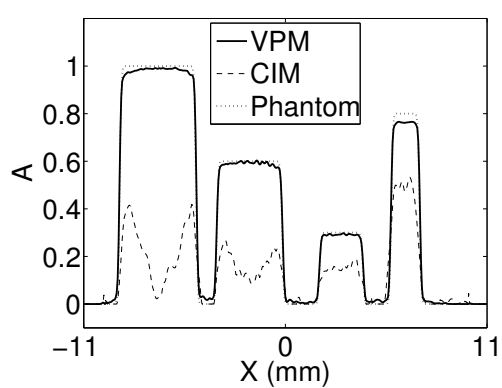

(c)

Figure 2: (a) and (b) Images reconstructed from noiseless data using the conventional iterative method (CIM) with $\lambda=1.0 \times 10^{-4}$ and the VP method (VPM) with $\lambda=1.0 \times 10^{-4}$ and $\alpha=1000$, respectively. (c) Image profiles through noiseless images seen in Fig. 2 corresponding to the reconstructions with the VP method (solid line), with the conventional iterative method (dashed line), and phantom (dotted line). The locations of the profiles are indicated by the " $\mathrm{X}$ " arrows in the Fig. 2a, and $2 \mathrm{~b}$, respectively.

comparing with the numerical phantom in Fig. 1a, the use of an inaccurate EIR can result in strong artifacts and distortions in images reconstructed by use of the conventional methods (RMSE $=0.1552$ ). As revealed by Fig. $2 \mathrm{~b}$ and the profiles in $2 \mathrm{c}$, the VP algorithm yielded an image with fewer artifacts and distortions, and the 


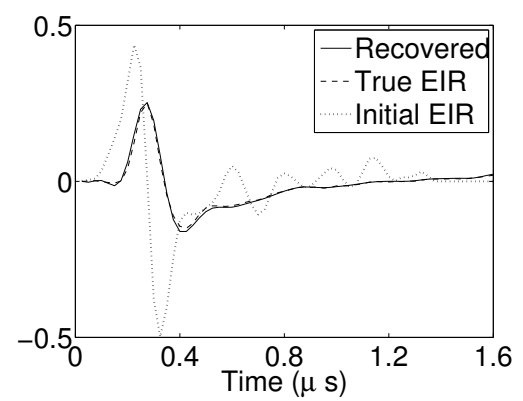

Figure 3: Recovered EIR

image fidelity was improved as reflected by the reduced RMSE of 0.0084 . The accuracy of the recovered EIR was also improved as indicated by Fig. 3 .

Figure 4 shows the images reconstructed from the noisy data and their profiles. The RMSEs are 0.1601 and 0.0283 for the conventional iterative method and VP method, respectively. These preliminary results suggest that the proposed joint reconstruction method is numerically stable.

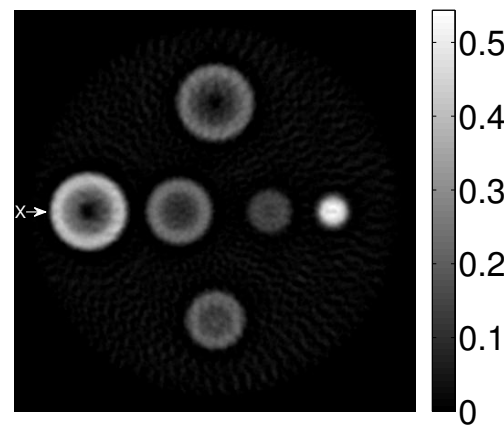

(a)

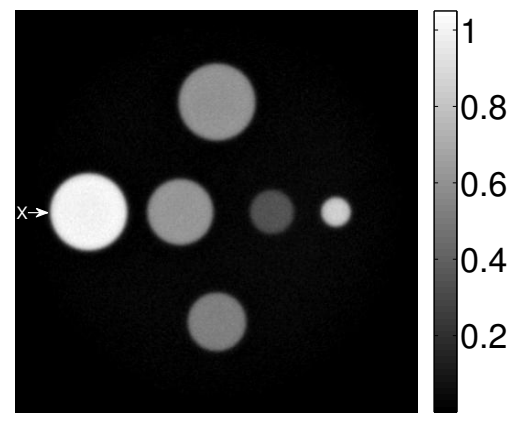

(b)

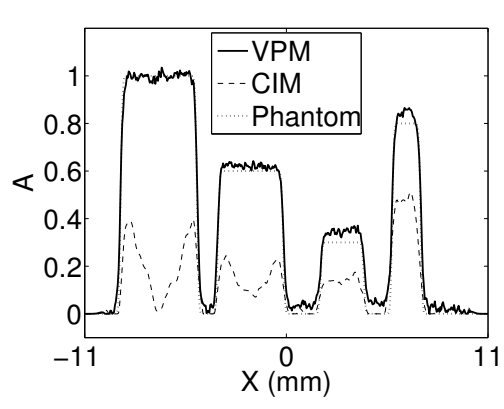

(c)

Figure 4: Images reconstructed from noise data using (a) the conventional iterative method with $\lambda=5.0 \times 10^{-3}$ and (b) the VP algorithm with $\lambda=5.0 \times 10^{-3}$ and $\alpha=10000$. (c) Image profiles through noiseless images seen in Fig. 4 corresponding to the reconstructions with the VP method (solid line), with the conventional iterative method (dashed line), and phantom (dotted line). The locations of the profiles are indicated by the "X" arrows in the Fig. $4 \mathrm{a}$, and $4 \mathrm{~b}$, respectively.

\section{SUMMARY}

It is known that neglecting or employing a contaminated EIR in PACT imaging reconstruction can result in distortions and artifacts in the reconstructed images. However, accurate measuring or modeling EIR can be difficult. In this study, we proposed a joint reconstruction approach for PACT that mitigates artifacts in the reconstructed images caused by use of an inaccurate EIR. A nonlinear least squares minimization problem was formulated, which exploited the separable nature of the imaging model, and a VP method was employed to solve the minimization problem. The results demonstrate that the joint reconstruction approach for estimating both the EIR and the absorbed optical energy density can increase the fidelity of the reconstructed images.

\section{ACKNOWLEDGMENTS}

This work was supported in part by NIH awards CA167446 and EB01696301. 


\section{REFERENCES}

1. Oraevsky, A. A. and Karabutov, A. A., [Optoacoustic Tomography], Biomedical Photonics Handbook, CRC Press (2003).

2. Wang, L. V., "Tutorial on photoacoustic microscopy and computed tomography," Selected Topics in Quantum Electronics, IEEE Journal of 14, 171-179 (Jan 2008).

3. Kruger, R. A., Reinecke, D. R., and Kruger, G. A., "Thermoacoustic computed tomographytechnical considerations," Medical Physics 26(9) (1999).

4. Cox, B. T., Arridge, S. R., Köstli, K. P., and Beard, P. C., "Two-dimensional quantitative photoacoustic image reconstruction of absorption distributions in scattering media by use of a simple iterative method," Applied Optics 45, 1866-1875 (Mar 2006).

5. Wang, K. and Anastasio, M. A., "Photoacoustic and thermoacoustic tomography: Image formation principles," in [Handbook of Mathematical Methods in Imaging], Scherzer, O., ed., Springer (2011).

6. Anastasio, M. A., Zhang, J., Modgil, D., and La Riviere, P., "Application of inverse source concepts to photoacoustic tomography," Inverse Problems 23, S21-S35 (2007).

7. Wang, K., Ermilov, S., Su, R., Brecht, H. P., Oraevsky, A., and Anastasio, M., "An imaging model incorporating ultrasonic transducer properties for three-dimensional optoacoustic tomography," Medical Imaging, IEEE Transactions on 30, 203-214 (Feb 2011).

8. Rosenthal, A., Ntziachristos, V., and Razansky, D., "Model-based optoacoustic inversion with arbitraryshape detectors," Medical Physics 38(7) (2011).

9. Wang, K., Su, R., Oraevsky, A. A., and Anastasio, M. A., "Investigation of iterative image reconstruction in three-dimensional optoacoustic tomography," Physics in Medicine and Biology 57(17), 5399 (2012).

10. Rosenthal, A., Ntziachristos, V., and Razansky, D., "Optoacoustic methods for frequency calibration of ultrasonic sensors," Ultrasonics, Ferroelectrics and Frequency Control, IEEE Transactions on 58, 316-326 (February 2011).

11. Chung, J. and Nagy, J., "An efficient iterative approach for large-scale separable nonlinear inverse problems," SIAM Journal on Scientific Computing 31(6), 4654-4674 (2010).

12. Aravkin, A. Y. and van Leeuwen, T., "Estimating nuisance parameters in inverse problems," Inverse Problems 28(11), 115016 (2012).

13. Golub, G. and Pereyra, V., "The differentiation of pseudo-inverses and nonlinear least squares problems whose variables separate," SIAM Journal on Numerical Analysis 10(2), 413-432 (1973).

14. Xu, M. and Wang, L. V., "Photoacoustic imaging in biomedicine," Review of Scientific Instruments $\mathbf{7 7}(4)$, $-(2006)$.

15. Wang, L. V., ed., [Photoacoustic Imaging and Spectroscopy], Optical Science and Engineering, CRC Press (March 2009). 